\title{
Deep Learning Enabled Measurements of Single-Atom Defects in 2D Transition Metal Dichalcogenides with Sub-Picometer Precision
}

Chia-Hao Lee ${ }^{1}$, Chuqiao Shi ${ }^{1}$, Di Luo ${ }^{2}$, Abid Khan ${ }^{2}$, Blanka E. Janicek ${ }^{1}$, Sangmin Kang ${ }^{3}$, Wenjuan $\mathrm{Zhu}^{3}$, Bryan K. Clark ${ }^{2}$ and Pinshane Y. Huang ${ }^{1 *}$

1. Department of Materials Science and Engineering, University of Illinois Urbana-Champaign, Urbana, IL, USA.

2. Department of Physics, University of Illinois Urbana-Champaign, Urbana, IL, USA.

3. Department of Electrical and Computer Engineering, University of Illinois Urbana-Champaign, Urbana, IL, USA.

* Corresponding author: pyhuang@illinois.edu

Pushing the limits of electron microscopy requires both technical development in instrumentation and the utilization of new approaches for data extraction. In our work, we apply deep learning techniques based on convolutional neural networks $(\mathrm{CNN})$, which have already revolutionized image recognition in fields such as medical diagnosis, weather forecasting, and facial recognition, to atomic-resolution images obtained using aberration-corrected scanning transmission electron microscopy (STEM) [1]. By utilizing the strong pattern recognition ability and adaptability of $\mathrm{CNN}$, we provide new opportunities to access the underlying information in large volumes of atomic-resolution images of two-dimensional transition metal dichalcogenides (2D TMDCs), whose properties have been strongly influenced by atomic defects such as vacancies and substitutional dopants. Yet, high-precision characterization of single-atom defects remains challenging because 2D materials are irradiation sensitive, produce low signals, and require low-voltage imaging modes. Typical imaging modes struggle to provide sufficiently high signal-to-noise ratio (SNR) and resolution to measure the structure of single-atom defects without inducing electron beam damage. Here, we utilize CNN to identify atomic defects in 2D TMDCs such as Mo ${ }_{1-\mathrm{x}} \mathrm{W}_{\mathrm{x}} \mathrm{Te}_{2}$ and $\mathrm{WSe}_{2-\mathrm{x}} \mathrm{Te}_{\mathrm{x}}$ and use the resulting data to tackle the above-mentioned characterization challenges in $2 \mathrm{D}$ materials. Using these techniques, we are able to measure local lattice strains induced by substitutional dopants with subpicometer precision, which have been traditionally difficult to extract without the aid of CNN.

First, we construct CNN models to identify and classify various defects, including metal substitutions, chalcogen vacancies, and chalcogen. Using the output of the CNN, we generate class-averaged images of each defect type using rigid-registration and averaging hundreds of nominally identical single-atom defects (Figure 1). These methods result in final images with high SNR, which improves approximately with the square root of the number of images summed [2]. The class-averaged images also enable highprecision measurements of the defect structure which are inaccessible in the raw data. Using 2D Gaussian fits, we compare the projected bond lengths at regular lattice and defect sites. We find $\mathrm{W}$ substitutions in $\mathrm{Mo}_{1-\mathrm{x}} \mathrm{W}_{\mathrm{x}} \mathrm{Te}_{2}$ decrease the projected Te-Te bond length by $1.7 \mathrm{pm}$, while Te substitutions in $\mathrm{WSe}_{2-\mathrm{x}} \mathrm{Te}_{\mathrm{x}}$ increase the projected W-W bond length by $2.4 \mathrm{pm}$. Importantly, we apply a bootstrapping method to determine the precision of our bond length measurements. In the $\mathrm{WSe}_{2-\mathrm{x}} \mathrm{Te}_{\mathrm{x}}$ system, we obtain standard deviation of $0.36 \mathrm{pm}$ in the class-averaged images, a nearly 30 -fold improvement when compared with the $10.6 \mathrm{pm}$ precision of the original images (Figure 2). In summary, we have developed measurement techniques using CNN to locate, classify, and measure the local structure of single-atom defects in 2D TMDCs with sub-picometer precision [3]. 
References:

[1] M Ziatdinov et al., ACS Nano 11 (2017), p. 12742.

[2] B Savitzky, et al., Ultramicroscopy 191 (2018), p. 56.

[3] This work was supported by the AFOSR under award number FA9550-7-1-0213 and carried out in part in the Materials Research Laboratory at UIUC. S.K. and W.Z. would like to acknowledge the support from ONR under grant NAVY N00014-17-1-2973.

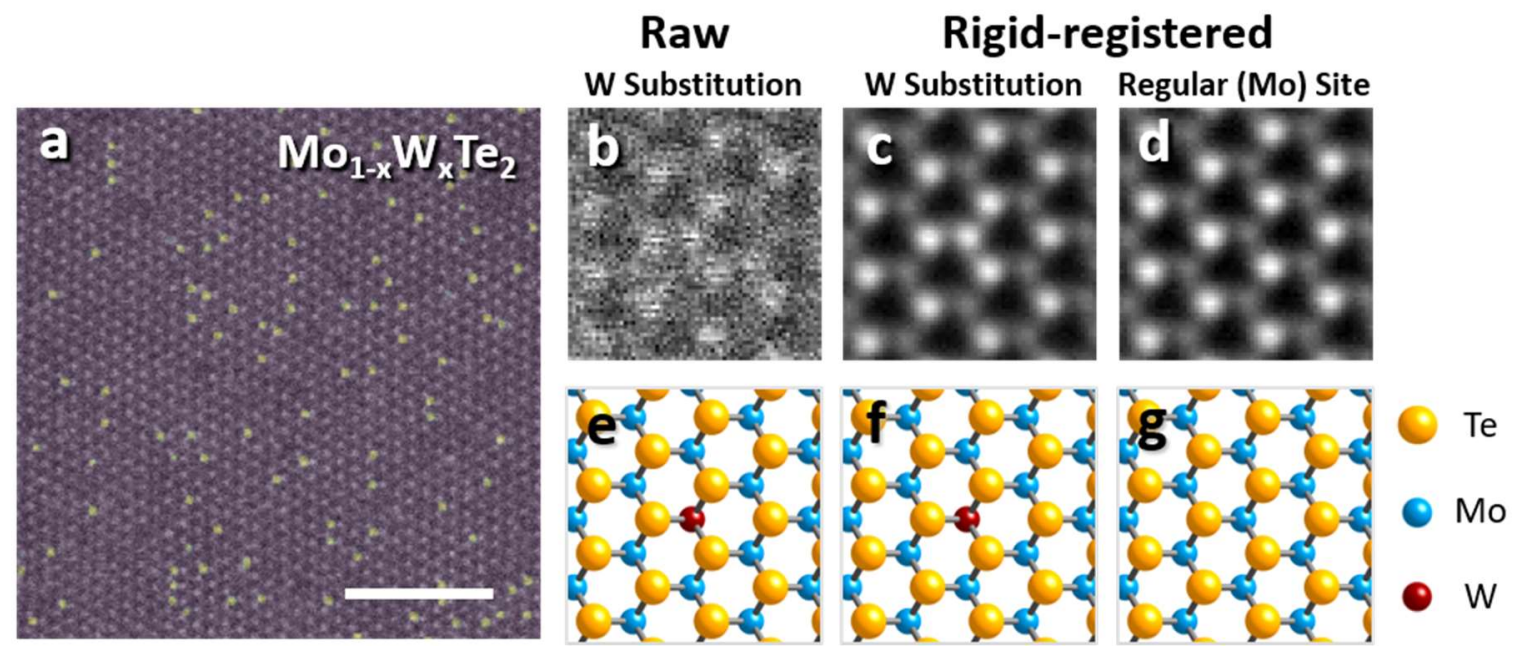

Figure 1. Identification of $\mathrm{W}$ substitutions using deep learning on a STEM image of $\mathrm{Mo}_{1-\mathrm{x}} \mathrm{W}_{\mathrm{x}} \mathrm{Te}_{2}$ (a) annular dark-field STEM image of $\mathrm{Mo}_{1-\mathrm{x}} \mathrm{W}_{\mathrm{x}} \mathrm{Te}_{2}$, overlaid with $\mathrm{CNN}$ determined positions of $\mathrm{W}$ substitutions in yellow and regular lattice sites in purple. Scale bar is $2.5 \mathrm{~nm}$. (b-d) Raw and summed images generated from (a) and (e-g) corresponding cartoons of regular Mo and W-substituted lattice sites. By aligning and summing 274 equivalent lattice positions, we produce rigid-registered images (c-d) with 15 times higher SNR than the raw image (b). Image widths in (b-d) are $1.3 \mathrm{~nm}$.
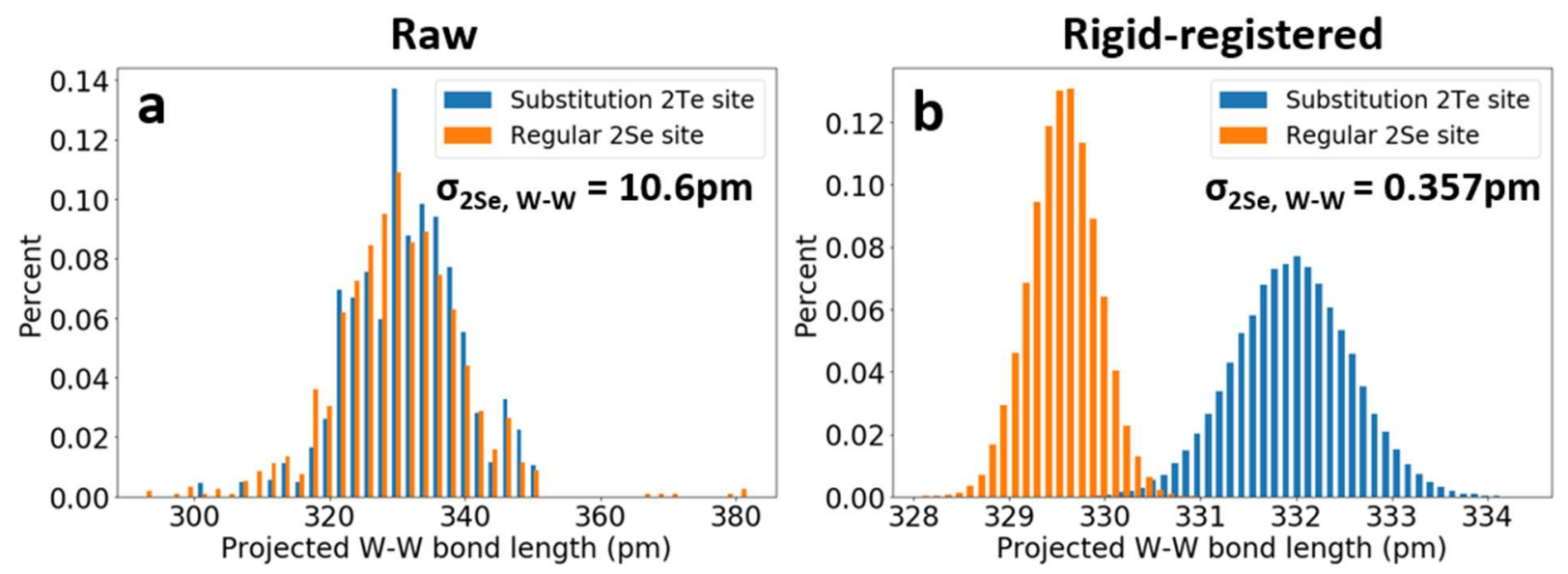

Figure 2. Distribution of projected $\mathrm{W}-\mathrm{W}$ bond length measurements of $\mathrm{WSe}_{2-\mathrm{x}} \mathrm{Te}_{\mathrm{x}}$ for (a) raw (b) rigidregistered images. Unlike in the raw data, the rigid-registered images show well-separated distributions of bond length measurements on regular and defect-containing (2Te-substituted) sites. The measured bond length of 2Te-substiuted site is $332.0 \pm 0.61 \mathrm{pm}$, and $329.6 \pm 0.36 \mathrm{pm}$ for the regular $2 \mathrm{Se}$ site. We are able to improve our precision by factor of 30 after aligning and summing images of 2Te (183 images) and 2Se (523 images) sites respectively. With these methods, we were able to measure the local strains in 2D materials induced by individual atomic substitutions with sub-picometer precision for the first time. 